\title{
MATHEMATICAL MODEL IN A THIN NON-HOMOGENEOUS ROTATING DISC FOR ISOTROPIC MATERIAL WITH RIGID SHAFT BY USING SETH'S TRANSITION THEORY
}

\author{
Pankaj Thakur', Jatinder Kaur ${ }^{2}$, Satya Bir Singh ${ }^{2}$ \\ ${ }^{1}$ Department of Mathematics, IEC University Baddi, Solan, Himachal Pradesh 174103, India \\ ${ }^{2}$ Department of Mathematics, Punjabi University Patiala, Punjab 147002, India \\ E-mails: dr_pankajthakur@yahoo.com, sbsingh69@yahoo.com
}

(Received April 22, 2014)

\begin{abstract}
In this paper we investigate stresses and displacement in thin nonhomogeneous rotating disc has been derived by using Seth's Transition theory and results have been discussed and depicted graphically. Non-homogeneity is assumed due to the variation of modulus of rigidity. As a numerical example, it has been seen that in the presence of non-homogeneity having values $k>0$ at the bore, reduces stresses, displacement and the angular speed as compare to lesser value i.e. $k<0$ of nonhomogeneity. Radial stresses maximum at the internal surface.
\end{abstract}

Keywords: Stresses, displacement, non-homogeneous, disc, angular speed, yielding.

\section{INTRODUCTION}

Rotating discs are historically of interest to designers due to their vast spectrum of use in the aerospace industry. Gas turbine discs are an important example of such applications. In turbojet engines, rotating discs are simultaneously subjected to mechanical and thermal loads. A disc may be under internal pressure due to shrink fit on its mounting shaft; in addition an external tensile load may be applied to its outer edge resulting from the blade effects installed on its outer periphery. Rotating Discs form an essential part of the design of rotating machinery, namely rotors, turbines, compressors, flywheel and computer's disc drive etc. Helicopter rotor blades are typically built-up composite structure and made of material that may be anisotropic and non-homogeneous. For wide class of materials such as hot rolled copper, aluminum and magnesium alloys some degree of non-homogeneity is presents. OLSZAK et al. [1] solved the problems of thick walled cylinder, non-homogeneous both elastically and plastically subjected to internal and external pressures and showed that plastic flow may start from either surface depending on the character and intensity of the nonhomogeneity. GHOSH [2] on the problem involving the study of elastic-plastic stress in a spherically pressure vessel of non-homogeneous material and MUKHOPADHYAY [3 ] studied the effect of non-homogeneity on yields stress in a thick walled cylinder tube under pressure. Gupta et al. [4] solved the problems effect of non-homogeneity on elastic-plastic transition in 
a thin rotating disc by using Seth's transition theory and plane stress condition. This theory [5] does not required any assumptions like an yield condition, incompressibility condition and thus poses and solves a more general problem from which cases pertaining to the above assumptions can be worked out. It utilizes the concept of generalized strain measure and asymptotic solution at critical points or turning points of the differential equations defining the deformed field and has been successfully applied to a large number of problems [7-12, 1443]. SETH [5] has defined the generalized principal strain measure as:

$e_{i i}=\int_{0}^{e_{i i}}\left[1-2 e_{i i}^{A}\right]^{\frac{n}{2}-1} d e_{i i}^{A}=\frac{1}{n}\left[1-\left(1-2 e_{i i}^{A}\right)^{\frac{1}{n}}\right],(i=1,2,3)$

where $n$ is the measure and $e_{i i}^{A}$.is the Almansi finite strain components. For $n=-2,-1,0,1,2$, it gives Cauchy, Green Hencky, Swainger and Almansi measures, respectively. The rigidity modulus of a thin rotating disc is assumed to vary radially i.e.

$\mu=\mu_{0} r^{-k}$

where $\mu_{0}$ and $k$ are real constants. In this research paper, we discuss mathematical model in a thin non-homogeneous rotating disc for isotropic material with shaft by using Seth's transition theory. Result obtained have been numerically and depicted graphically.

\section{MATHEMATICAL MODELS AND GOVERNING EQUATION}

We consider an annular disc of inner radius $a$ and outer radius $b$ rotating with an angular speed $\omega$ about an axis perpendicular to its plane and passed through the center as shown in Figure 1. The annular disc is mounted on a rigid shaft. The disc is made of the material having constant density $\rho$ but variable modulus of rigidity $\mu=\mu(r)$ and thickness of disc is assumed sufficiently small so that it is effectively in a state of plane stress, that is, the axial stress $T_{z z}$ is zero.

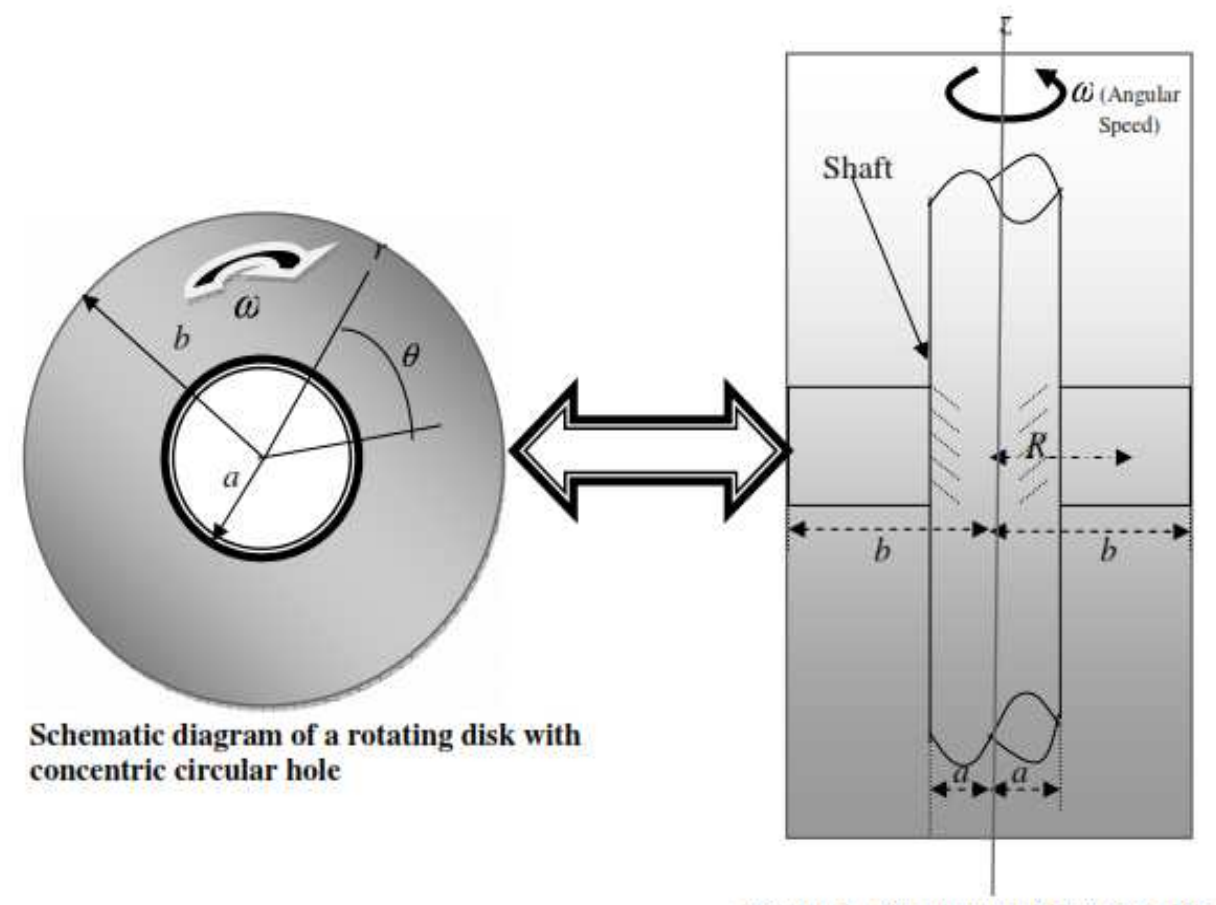

Figure 1. Geometry of Rotating Disc. 
The displacement components in cylindrical polar co- ordinate are given by [6]:

$u=r(1-\beta), v=0, w=d z$.

where $\beta$ is function of $r=\sqrt{x^{2}+y^{2}}$ only and $d$ is a constant.

The finite strain components are given by Seth [6] as:

$$
\begin{aligned}
& \stackrel{A}{e}_{r r} \equiv \frac{\partial u}{\partial r}-\frac{1}{2}\left(\frac{\partial u}{\partial r}\right)^{2}=\frac{1}{2}\left[1-\left(r \beta^{\prime}+\beta\right)^{2}\right], e_{\theta \theta}^{A} \equiv \frac{u}{r}-\frac{u^{2}}{2 r^{2}}=\frac{1}{2}\left[1-\beta^{2}\right], \\
& \stackrel{A}{e}_{z z} \equiv \frac{\partial w}{\partial z}-\frac{1}{2}\left(\frac{\partial w}{\partial z}\right)^{2}=\frac{1}{2}\left[1-(1-d)^{2}\right], e_{r \theta}^{A}=\stackrel{A}{e_{\theta \varepsilon}}=\stackrel{A}{e_{z r}}=0 .
\end{aligned}
$$

where $\beta^{\prime}=d \beta / d r$.

Substituting equation (4) in equation (1), the generalized components of strain are:

$$
e_{r r}=\frac{1}{n}\left[1-\left(r \beta^{\prime}+\beta\right)^{n}\right], e_{\theta \theta}=\frac{1}{n}\left[1-\beta^{n}\right], e_{z z}=\frac{1}{n}\left[1-(1-d)^{n}\right], e_{r \theta}=e_{\theta z}=e_{z r}=0
$$

where $r \beta^{\prime}=\beta P$.

The stress -strain relations for isotropic material are given by [13]:

$T_{i j}=\lambda \delta_{i j} I_{1}+2 \mu e_{i j}(i, j=1,2,3)$,

where $T_{i j}$ and $e_{i j}$ are the stresses and strain components, $\lambda$ are lame's constant, $\mu=\mu(r)$, $I_{1}=e_{k k}$ is the first strain invariant, $\delta_{i j}$ is the Kroncecker's delta.

Equations (6) for this problem become:

$$
T_{r r}=\frac{2 \lambda \mu}{\lambda+2 \mu}\left[e_{r r}+e_{\theta \theta}\right]+2 \mu e_{r r}, T_{\theta \theta}=\frac{2 \lambda \mu}{\lambda+2 \mu}\left[e_{r r}+e_{\theta \theta}\right]+2 \mu e_{\theta \theta}, T_{z z}=T_{r \theta}=T_{\theta z}=T_{z r}=0
$$

where $\lambda$ is a constant and $\mu=\mu(r)$.

Using equation (4) in equation (6), the strain components in terms of stresses are obtained as [16]:

$$
\begin{aligned}
& e_{r r}=\frac{\partial u}{\partial r}-\frac{1}{2}\left(\frac{\partial u}{\partial r}\right)^{2}=\frac{1}{2}\left[1-\left(r \beta^{\prime}+\beta\right)^{2}\right]=\frac{1}{E}\left[T_{r r}-\left(\frac{1-C}{2-C}\right) T_{\theta \theta}\right], \\
& e_{\theta \theta}=\frac{u}{r}-\frac{u^{2}}{2 r^{2}}=\frac{1}{2}\left[1-\beta^{2}\right]=\frac{1}{E}\left[T_{\theta \theta}-\left(\frac{1-C}{2-C}\right) T_{r r}\right], \\
& e_{z z}=\frac{\partial w}{\partial z}-\frac{1}{2}\left(\frac{\partial w}{\partial z}\right)^{2}=\frac{1}{2}\left[1-(1-d)^{2}\right]=-\frac{(1-C)}{E(2-C)}\left[T_{r r}-T_{\theta \theta}\right], e_{r \theta}^{A}=e_{\theta z}^{A}=e_{z r}^{A}=0 .
\end{aligned}
$$

where $E=\frac{\mu(3 \lambda+2 \mu)}{(\lambda+\mu)}$ and $C=\frac{2 \mu}{\lambda+2 \mu}$.

Substituting equation (5) in equation (7), we get the stress as:

$$
\begin{aligned}
& T_{r r}=\frac{2 \mu}{n}\left[3-2 C-\beta^{n}\left\{1-C+(2-C)(P+1)^{n}\right\}\right], T_{\theta \theta}=\frac{2 \mu}{n}\left[3-2 C-\beta^{n}\left\{2-C+(1-C)(P+1)^{n}\right\}\right], \\
& T_{r \theta}=T_{\theta z}=T_{z r}=T_{z z}=0
\end{aligned}
$$

where $r \beta^{\prime}=\beta P$. Equations of equilibrium are all satisfied except: 
$\frac{d}{d r}\left(r T_{r r}\right)-T_{\theta \theta}+\rho \omega^{2} r^{2}=0$

where $\rho$ is the density of the material of the rotating disc.

Using equation (9) and (10), we get a non- linear differential equation in $\beta$ as:

$n(2-C) \mu \beta^{n+1} P(P+1)^{n-1} \frac{d P}{d \beta}=\left[\begin{array}{l}r \mu^{\prime}\left[3-2 C-\beta^{n}\left\{1-C+(2-C)(P+1)^{n}\right\}\right] \frac{n \rho \omega^{2} r^{2}}{2 \mu} \\ -\mu\left[2 r C^{\prime}-C^{\prime} r \beta^{n}\left\{1+(P+1)^{n}\right\}+n \beta^{n} P\left\{1-C+(2-C)(P+1)^{n}\right\}\right] \\ +\mu \beta^{n}\left[1-(P+1)^{n}\right]\end{array}\right]$

where $\beta^{\prime}=d \beta / d r$ ( $P$ is function of $\beta$ and $\beta$ is function of $r$ only). From equation (11), the transition points of $\beta$ are $P=-1$ and $\pm \infty$.

Boundary condition: The boundary conditions are given by:

$u=0$, at $r=a, T_{r r}=0, a t r=b$.

where $T_{r r}$ are the radial stresses and $u$ are the displacement.

\section{SOLUTION THROUGH THE PRINCIPAL STRESSES}

For finding the plastic stress, the transition function is taken through the principal stress (see SeTh [5, 6], Hulsurkar [7], Gupta etl. [4, 10- 12], Shukla [8, 9 ], Thakur $[14-42])$ at the transition point $P \rightarrow \pm \infty$. We take the transition function $T$ is defined as:

$T=\frac{n T_{\theta \theta}}{2 \mu}=\left[(3-2 C)-\beta^{n}\left\{2-C+(1-C)(P+1)^{n}\right\}\right]$

where $T$ is function of $r$ only. Taking the logarithmic differentiation of equation (13) with respect to $r$, we get:

$$
\frac{d(\log T)}{d r}=-\left[\frac{+n(1-C) \beta^{n+1} P(P+1)^{n-1} d P / d \beta}{r\left[3-2 C-\beta^{n}\left\{\begin{array}{l}
2-C \\
\left.+(1-C)(P+1)^{n}\right\}
\end{array}\right]\right.}\right]+\frac{\mu^{\prime}}{\mu}
$$

Substituting the value of $d P / d \beta$ from equation (11) in equation (14) and taking the asymptotic value as $P \rightarrow \pm \infty$, we get after integration:

$T=A\left(\frac{1-C}{2-C}\right) \exp f(r)$.

where $f(r)=-\int[1 / r(2-C)] d r$ and $A$ is a constant of integration and can be determined by the given boundary condition 
From equations (13) and (15), we have:

$$
T_{\theta \theta}=A\left(\frac{1-C}{2-C}\right) \exp f(r)
$$

Substituting equation (16) in equation (10) and integrating, we get:

$$
r T_{r r}=B+A \int I(r) d r-\frac{\rho \omega^{2} r^{3}}{3}
$$

where $B_{1}$ is a constant of integration and can be determined by the given boundary condition and $I(r)=\left(\frac{1-C}{2-C}\right) \exp f(r)$.

Substituting equations (16) and (17) in second equation of equation (8), we get:

$$
\beta=\sqrt{1-\frac{2}{E}\left(\frac{1-C}{2-C}\right)\left[A\left(\exp f(r)-\frac{1}{r} \int I(r) d r\right)+\frac{\rho \omega^{2} r^{2}}{3}-\frac{B}{r}\right]}
$$

Substituting equation (18) in equation (3), we get

$$
u=r-r \sqrt{1-\frac{2}{E}\left(\frac{1-C}{2-C}\right)\left[A\left(\exp f(r)-\frac{1}{r} \int I(r) d r\right)+\frac{\rho \omega^{2} r^{2}}{3}-\frac{B}{r}\right]}
$$

where $E=2 \mu(3-2 C) /(2-C)$ is the Young's modulus.

Using boundary condition (12 in equations (17) and (19), we get the values of constant of integration:

$$
A=\frac{\rho \omega^{2}\left(b^{3}-a^{3}\right)}{3\left\{[a \exp . f(r)]_{r=a}+\int_{a}^{b} I(r) d r\right\}} \text { and } B=\frac{\rho \omega^{2} b^{3}}{3}-\frac{\rho \omega^{2}\left(a^{3}-b^{3}\right) \int^{b} I(r) d r}{3\left\{[a \exp . f(r)]_{r=a}+\int_{a}^{b} I(r) d r\right\}} \text {. }
$$

Substituting the values of constant of integration $A$ and $B$ from equations (16), (17), and (19) respectively, we get the transitional stresses and displacement as:

$$
\begin{aligned}
& T_{\theta \theta}=\frac{\rho \omega^{2}\left(b^{3}-a^{3}\right)}{3\left\{[a \exp . f(r)]_{r=a}+\int_{a}^{b} I(r) d r\right\}} I(r), T_{r r}=\frac{\rho \omega^{2}}{3 r}\left[b^{3}-r^{3}+\frac{\left(b^{3}-a^{3}\right) \int_{r}^{b} I(r) d r}{\left.\left\{[a \exp \cdot f(r)]_{r=a}+\int_{a}^{b} I(r) d r\right\}\right]}\right. \\
& u=r-r \sqrt{1-\frac{2}{E}\left(\frac{1-C}{2-C}\right) \frac{\rho \omega^{2}}{3 r}\left[r^{3}-b^{3}+\frac{\left.\left(b^{3}-a^{3}\right)\left\{r \cdot \exp f(r)+\int_{r}^{b} I(r) d r\right\}\right]}{\left\{[a \exp \cdot f(r)]_{r=a}+\int_{a}^{b} I(r) d r\right\}}\right]} .
\end{aligned}
$$

Substituting eqn. (2) in equation (20), we get: 


$$
\begin{aligned}
& T_{\theta \theta}=\frac{\rho \omega^{2}\left(b^{3}-a^{3}\right)\left(r^{k}+\mu_{0} / \lambda\right)^{\frac{1}{2 k}-1}}{6 r^{1-k}\left(b^{k}+\mu_{0} / \lambda\right)^{\frac{1}{2 k}}}, T_{\theta \theta}=\frac{\rho \omega^{2}}{3 r}\left[\frac{\left(b^{3}-a^{3}\right)\left\{\left(r^{k}+\mu_{0} / \lambda\right)^{\frac{1}{2 k}}\right\}_{r}^{b}}{\left(b^{k}+\mu_{0} / \lambda\right)^{\frac{1}{2 k}}}-r^{3}+b^{3}\right] \\
& u=r-r \sqrt{1-\frac{\rho \omega^{2} r^{2 k}\left(r^{3}-a^{3}\right)}{3 r \mu_{0}\left[3 r^{k}+2\left(\mu_{0} / \lambda\right)\right]}} .
\end{aligned}
$$

Initial yielding. From equation (21), it is seen that $\left|T_{r r}-T_{\theta \theta}\right|$ is maximum at the internal surface (that is at $r=a$ ), therefore yielding will take place at the internal surface of the disc and equation (21) are becomes:

$\left|T_{r r}-T_{\theta \theta}\right|_{r=a}=\left|\frac{\rho \omega^{2}\left(b^{3}-a^{3}\right)}{3 a}\left\{2-\left(\frac{a^{k}+\mu_{0} / \lambda}{b^{k}+\mu_{0} / \lambda}\right)^{\frac{1}{2 k}}\left(\frac{2\left(a^{k}+\mu_{0} / \lambda\right)+a^{k}}{2\left(a^{k}+\mu_{0} / \lambda\right)}\right)\right\}\left(\frac{a}{b}\right)^{1-C / 2-C}\right| \equiv Y$ (say).

angular velocity $\omega_{i}$ required for initial yielding is given by:

$$
\Omega_{i}^{2}=\frac{\rho \omega_{i}^{2} b^{2}}{Y}=\frac{3 a b^{2}}{\left(b^{3}-a^{3}\right)\left\{2-\left(\frac{a^{k}+\mu_{0} / \lambda}{b^{k}+\mu_{0} / \lambda}\right)^{\frac{1}{2 k}}\left(\frac{3 a^{k}+\mu_{0} / \lambda}{2\left(a^{k}+\mu_{0} / \lambda\right)}\right)\right\}}
$$

and $\omega_{i}=\frac{\Omega_{i}}{b} \sqrt{\frac{Y}{\rho}}$. We introduce the following non-dimensional components as $R=r / b, R_{0}=$ $a / b, \sigma_{r}=T_{r r} / Y, \sigma_{\theta}=T_{\theta \theta} / Y, H=Y / \mu_{0}$, and $\bar{u}=u / b$. Equations (21) and (22) becomes:

$$
\begin{aligned}
& \sigma_{\theta}=\frac{\Omega_{i}^{2} R^{k-1}\left(1-R_{0}^{3}\right)}{6\left(R^{k}+N_{1}\right)} S, \sigma_{r}=\frac{\Omega_{i}^{2}}{3 R}\left[\left(1-R_{0}^{3}\right)(1-S)-R^{3}+1\right] \bar{u}=R-R \sqrt{1-\frac{\Omega_{i}^{2} H R^{2 k-1} b^{k}\left(R^{3}-R_{0}^{3}\right)}{3\left[3 R^{k}+2 N_{1}\right]}} \\
& \Omega_{i}^{2}=\frac{\rho \omega_{i}^{2} b^{2}}{Y}=\frac{3 R_{0}}{\left(1-R_{0}^{3}\right)\left\{2-S \cdot\left(\frac{3 R_{0}^{k}+N_{1}}{2\left(R_{0}^{k}+N_{1}\right)}\right)\right\}},
\end{aligned}
$$

where $S=\left(\frac{R^{k}+N_{1}}{1+N_{1}}\right)^{\frac{1}{2 k}}$ and $N_{1}=\mu_{0} b^{-k} / \lambda$.

Fully Plastic State. The angular speed $\omega_{f}\left(>\omega_{i}\right)$ at which the disc become fully plastic $(\lambda \rightarrow \infty)$ is obtained from equation (21) as:

$\left|T_{r r}-T_{\theta \theta}\right|_{r=b}=\left|\frac{\rho \omega^{2}\left(b^{3}-a^{3}\right)}{6 b}\right| \equiv Y^{*}$

The angular velocity $\omega_{f}$ for fully-plastic state is given by: 
$\Omega_{f}^{2}=\frac{\rho \omega_{f}^{2} b^{2}}{Y^{*}}=\left|\frac{6 b^{3}}{\left(b^{3}-a^{3}\right)}\right|$

where $\omega_{f}=\frac{\Omega_{f}}{b} \sqrt{\frac{Y^{*}}{\rho}}$.Stresses, displacement and angular speed for fully- plastic state $(\lambda \rightarrow \infty)$ in non dimensional form are obtained from equations (21) and (24) become:

$$
\begin{aligned}
& \sigma_{\theta}=\frac{\Omega_{f}^{2}}{6 R}\left[\left(1-R_{0}^{3}\right) R^{\frac{1}{2}}\right], \sigma_{r}=\frac{\Omega_{f}^{2}}{3 R}\left[\left(1-R_{0}^{3}\right)\left(1-R^{\frac{1}{2}}\right)-R^{3}+1\right], \\
& \bar{u}_{f}=R-R \sqrt{1-\frac{\Omega_{f}^{2} R^{k-1} b^{k}\left(R^{3}-R_{0}^{3}\right) H}{9}}, \Omega_{f}^{2}=\frac{\rho \omega_{f}^{2} b^{2}}{Y^{*}}=\left|\frac{6}{\left(1-R_{0}^{3}\right)}\right| .
\end{aligned}
$$

\section{NUMERICAL ILLUSTRATION AND DISCUSSION}

For calculating the stresses, angular speed and displacement based on the above analysis, the following values have been taken as $k=-1,0.5,2, E / Y=1,2$ and $N=\mu_{0} / \lambda=2$ respectively. We considered two types of discs as shown in Table 1.

- Discs having less non-homogeneity at the bore than at the rim, i.e. $k<0$.

- Discs having more non-homogeneity at the bore than at the rim, i.e. $k>0$.

It can be seen from Table 1, that disc having more non-homogeneity values $k>0$ at the bore than at the rim required higher percentage increase in angular speed to become fully plastic from its initial yielding as compared to rotating disc having lesser value of non-homogeneity $k<0$ at the bore. Curves have been drawn in fig. 1 between angular speed $\Omega_{i}^{2}$ required for initial yielding and various radii ratios $R_{0}=a / b$ for $k=-1,0.5,2$.

\begin{tabular}{|c|c|c|c|c|}
\hline | & $\begin{array}{l}\text { Non- } \\
\text { homogeneity } \\
\text { of disc } \\
k\end{array}$ & $\begin{array}{c}\text { Angular } \\
\text { Speed required } \\
\text { for initial } \\
\text { yielding } \\
\Omega_{i}^{2}\end{array}$ & $\begin{array}{c}\text { Angular Speed } \\
\text { required for } \\
\text { fully- plastic } \\
\text { state } \\
\Omega_{f}^{2}\end{array}$ & $\begin{array}{c}\text { Percentage } \\
\text { increase in } \\
\text { Angular speed } \\
\left(\sqrt{\frac{\Omega_{f}^{2}}{\Omega_{i}^{2}}}-1\right) \times 100\end{array}$ \\
\hline $\begin{array}{l}\stackrel{0}{-} \\
v \\
\simeq \\
v \\
v \\
n\end{array}$ & $\begin{array}{lc}-1 & (k<0) \\
-0.5 & (k<0) \\
0.5 & (k>0) \\
1 & (k>0) \\
2 & (k>0)\end{array}$ & $\begin{array}{l}1.99588204 \\
1.9658364 \\
1.901275326 \\
1.868495965 \\
1.806642201\end{array}$ & $\begin{array}{l}6.857143 \\
6.857143 \\
6.857143 \\
6.857143 \\
6.857143\end{array}$ & $\begin{array}{l}85.35494 \% \\
86.76604 \% \\
89.91055 \% \\
91.56913 \% \\
94.82089 \%\end{array}$ \\
\hline
\end{tabular}

Table 1: Angular speed required for initial yielding and fully plastic state

It has been observed that discs having less non-homogeneity (i.e. $k=-1$ ) require higher angular speed to yield at the internal surface as compare to disc having more nonhomogeneity at the bore than at the rim, i.e. $k=2$. In fig. 3 and 4 , curves have been drawn for stresses and displacement with respect to radii ratio $R=r / b$ for elastic-plastic transition and fully plastic state respectively. Discs having less non-homogeneity at the bore required 
maximum radial stresses and circumferential stresses as compared to disc have more nonhomogeneity at the bore than at the rim. It has been seen that radial stresses is maximum at the internal surface. Rotating disc likely fracture at the bore.

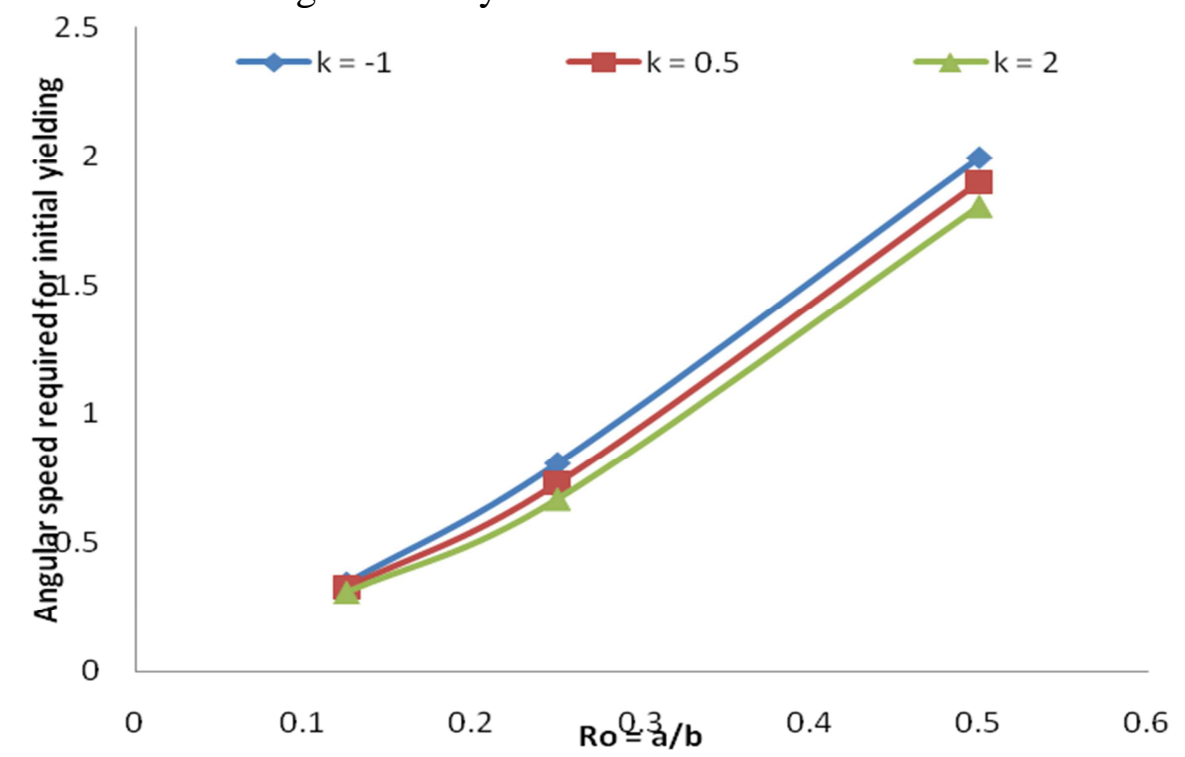

Figure 2. Angular speed required for initial yielding at the internal surface of the rotating disc with rigid inclusion along the radii ratio $R_{0}=a / b$ for $k=-1,0.5,2$.

Meaning: sigma theta $\sigma_{\theta}$ (circumferential stress); sigma r- $\sigma_{r}$ (Radial stress) and displacement- $U$

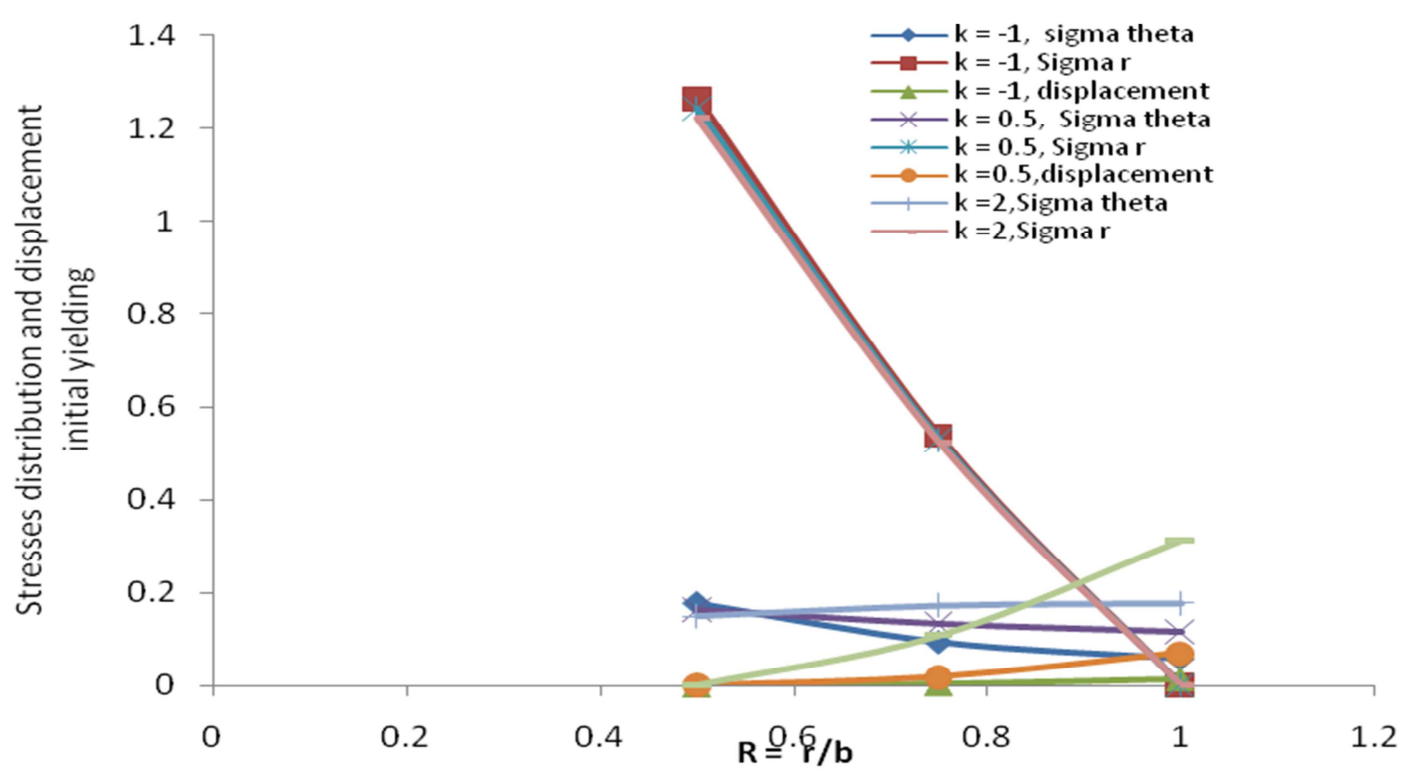

Figure 3. Stresses and Displacement at the elastic-plastic transition of rotating disc along the radius $\mathrm{R}=\mathrm{r} / \mathrm{b}$ 

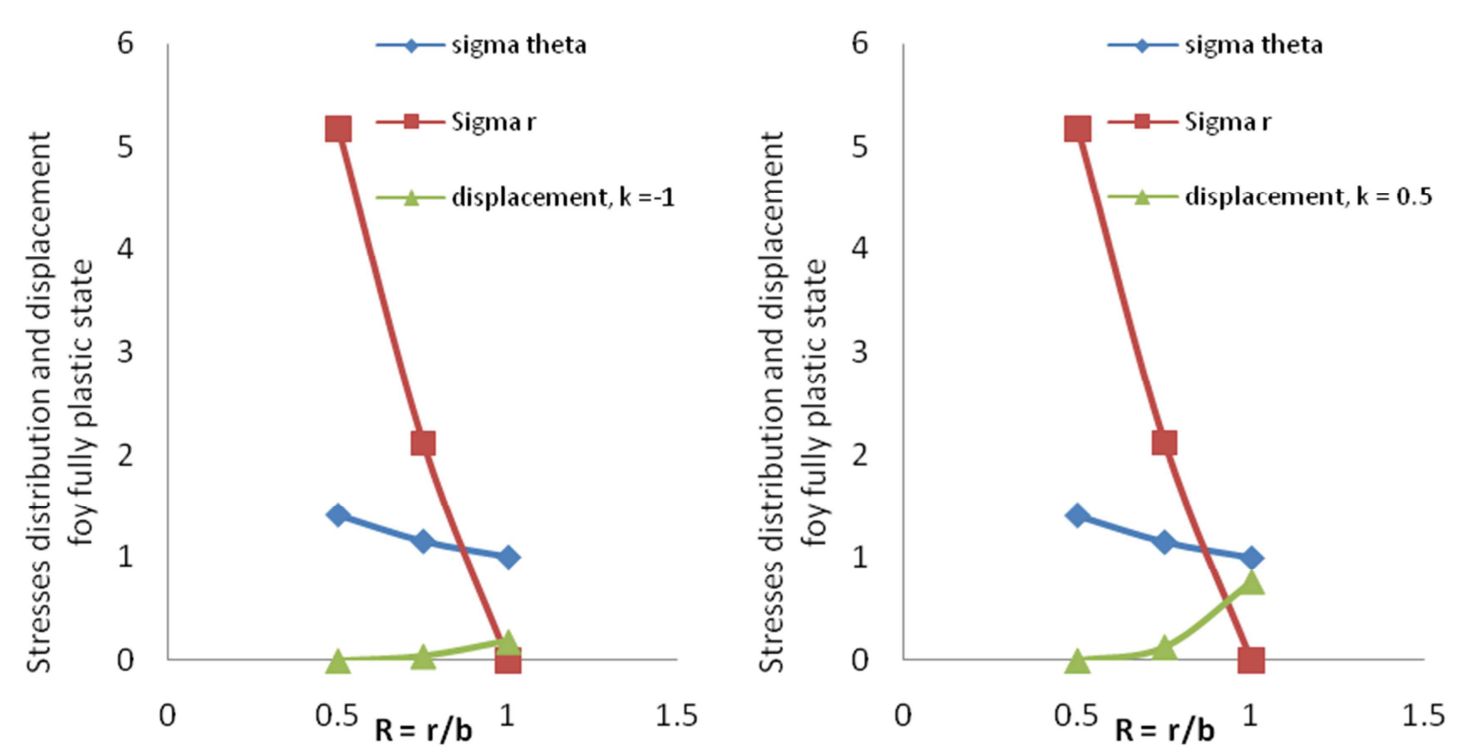

Figure 4. Stresses and Displacement for fully-plastic state of rotating disc along the radius $\mathrm{R}=r / b$.

\section{CONCLUSION}

It has been observed that discs having less non-homogeneity $(\mathrm{k}<0)$ require higher angular speed to yield at the internal surface as compare to disc having more nonhomogeneity at the bore than at the rim $(\mathrm{k}>2$ ). Discs having less non-homogeneity at the bore required maximum radial stresses and circumferential stresses as compared to disc have more non-homogeneity at the bore than at the rim. It has been seen that radial stresses is maximum at the internal surface.

\section{References:}

[1] OlSZAK, W., URBANOWSKI, W., Non-homogeneous Thick walled cylinder subjected to internal and external pressure, Arch. Mech. Stos. 3 (1955) 315-336.

[2] GHOsh, D., Study of elasticos-plastic stresses in a spherical pressure vessel of nonhomogeneous material, J. Sci. Engng. Resi. 7 (1963) 307-333.

[3] MukHOPADHYAY, J., Effect of non-homogeneity on yield stress in a thick walled cylindrical tube under pressure, Lett. Appl. Engng. Sci. 20 (1982) 963-968.

[4] GUPTA S.K., SHUKLA, R K., Effect of non-homogeneity on elastic-plastic transition in a thin rotating disk, Indian J. pure appl. Math. 25 (10) (1994) 1089-1097.

[5] SETH, B.R., Transition theory of Elastic-plastic Deformation, Creep and Relaxation, Nature 195 (1962) 896-897.

[6] SETH, B.R., Measure Concept in Mechanics, Int. J. Non-linear Mech. 1 (1) (1966) 3540.

[7] HULSURKAR, S., Transition theory of creep shells under uniform pressure, ZAMM, 46 (1) (1966) 431-437.

[8] SHUKLA, R.K., Creep transition in a thin rotating non-homogeneous disc, Indian Journal of pure and applied mathematics 27 (56) (1996) 487-498. 
[9] SHUKLA R.K., Elastic-Plastic transitional stresses in a non-homogeneous disc with variable thickness, subjected to internal pressure, Indian Journal of Pure and Applied Mathematics 31 (6) (2000) 713-720.

[10] GUPTA S.K , PANKAJ, Creep Transition in an isotropic disc having variable thickness subjected to internal pressure, Proc. Nat. Acad. Sci. India, Sect. A 78 (I) (2008) 57-66.

[11] GUPTA S.K., PANKAJ, Thermo elastic - plastic transition in a thin rotating disc with inclusion, Thermal Science 11 (1) (2007) 103-118.

[12] GUPTA S.K., PANKAJ, Creep transition in a thin rotating disc with rigid inclusion, Defence Science Journal 57 (2) (2007) 185-195.

[13] Sokolinikoff, I.S., Mathematical theory of Elasticity, Second edition, McGraw - Hill Book Co., New York (1950) 70-71.

[14] Pankaj Thakur, Some Problems in Elastic-plastic and Creep Transition, Ph.D. Thesis, Department of Mathematics, H.P.U. Shimla, India (2006).

[15] PANKaj ThakuR, Elastic-Plastic Transition Stresses in a transversely isotropic Thick walled Cylinder subjected to internal Pressure and steady - state Temperature, Thermal Science 13 (4) (2009) 107-118.

[16] Pankaj ThakUR Pankaj Thakur, Elastic-plastic transition stresses in a thin rotating disc with rigid inclusion by infinitesimal deformation under steady state Temperature, Thermal Science 14 (1) (2012) 209-219.

[17] PANKaJ ThaKuR, Creep Transition Stresses in a thin rotating disc with shaft by finite deformation under steady state temperature, Thermal Science, 14 (2) (2010) 425-436.

[18] Pankaj Thakur, Elastic - Plastic Transition Stresses In Rotating Cylinder By Finite Deformation Under Steady- State Temperature, Thermal Science 15 (2) (2011) 537-543.

[19] PANKaJ ThaKuR, Creep Transition stresses of a Thick isotropic spherical shell by finitesimal deformation under steady state of temperature and internal pressure, Thermal Science 15 (Suppl. 2) (2011) S157-S165.

[20] PANKaJ ThaKuR, Steady thermal stress and strain rates in a circular cylinder with nonhomogeneous compressibility subjected to thermal load, Thermal Science 18 (Suppl. 1) (2014) 81-92.

[21] PANKaJ ThaKUR, Steady thermal stress and strain rates in a rotating circular cylinder under steady state temperature, Thermal Science 18 (Suppl.1) (2014) 93-106.

[22] PANKaj ThakuR, Stresses in a thin rotating disc of variable thickness with rigid shaft, Journal for Technology of Plasticity 37 (1) (2012) 1-14.

[23] PANKAJ THAKUR, Elastic-plastic transition stresses in an isotropic disc having variable thickness subjected to internal pressure, International journal of Physical Science, African Journal 4 (5) (2009) 336-342.

[24] Pankaj Thakur, GauraV S., Creep transition stresses in thick walled rotating cylinder by finitesimal deformation under steady state temperature, International Journal of Mechanics and Solids, India 4 (1) (2009) 39-44.

[25] Pankaj ThaKUR, Elastic-Plastic Transition in a Thin Rotating Disc having variable density with Inclusion, Structural Integrity and life 9 (3) (2009) 171-179.

[26] PANKAJ ThakUR, Elastic-Plastic Transitional Stresses in a Thin Rotating Disc with Loading Edge, Proceeding of International conference on Advances in Modeling, 
optimization and Computing (AMOC-2011), Department of Mathematics, Indian Institute of Technology Roorkee, Roorkee, Dec. 5-7 (2011) 318-326.

[27] PANKaJ ThaKUR, Stresses in a spherical shell by using Lebesgue measure concept, International Journal of the Physical Sciences 6 (28) (2011) 6537-6540.

[28] PANKAJ THAKUR, Effect of transition stresses in a disc having variable thickness and Poisson's ratio subjected to internal pressure, WSEAS Transactions on Applied and Theoretical Mechanics 6 (4) (2011) 147-159.

[29] PANKaJ ThaKUR, Creep transition stresses in a spherical shell under internal pressure by using lebesgue measure concept, International journal Applied Mechanics and Engineering, Poland 16 (3) (2011) 83-87.

[30] Pankaj Thakur, Deformation in a thin rotating disc having variable thickness and edge load with inclusion at the elastic-plastic transitional stresses, Structural Integrity and life 12 (1) (2012) 65-70

[31] Pankaj Thakur, Thermo Creep Transition Stresses in a Thick-Walled Cylinder Subjected to Internal Pressure by finitesimal deformation, Structural Integrity and Life 12 (3) (2012) 165-173.

[32] PANKaj ThakuR, Effect of Stresses in a thin Rotating disk Loading Edge for different materials, International Journal for Technology of Plasticity 38 (1) (2013) 30-41.

[33] PANKaJ Thakur, Singh, S.B., JATINDER K., Thickness variation parameter in a thin rotating disc by finite deformation, FME Transaction 41 (2) (2013) 96-102.

[34] Pankaj Thakur, Analysis of Stresses in a Thin Rotating Disc With Inclusion and Edge Loading, Scientific Technical Review 63 (3) (2013) 9-16.

[35] PANKaj ThakuR, Stresses in a thick-walled circular cylinder having Pressure by using concept of generalized strain measure, Kragujevac Journal of Science 35 (2013) 41-48.

[36] Pankaj Thakur, SAndeEP K., Analysis of stresses in a Transversely Isotropic Thin Rotating Disc with rigid inclusion having variable density parameter, JSTP Open ejournal, Japan 9 (1) (2013) 1-13.

[37] Pankaj Thakur, Singh S. B., Jatinder K., Steady Thermal stresses in a rotating disk with shaft having density variation parameter subjected to thermal load, Structural Integrity and Life 13 (2) (2013) 109-116.

[38] PANKaJ ThakuR, Creep transitional stresses of Orthotropic Thick-Walled cylinder under combined axial Load under internal Pressure, Facta Universities Series: Mechanical engineering 11 (1) (2013) 13-18.

[39] PANKaj Thakur, Finitesimale deformation in a transversely isotropic Thin rotating disc with rigid shaft, International Journal for Technology of Plasticity 38 (2) (2013) 143-156.

[40] Jatinder Kaur, Pankaj Thakur, S.B. Singh, Steady thermal stresses in a thin rotating disc of finitesimal deformation with edge loading accepted for publication Journal of Solid Mechanics, Iran 7 (2) 2015.

[41] Pankaj Thakur, S. B. Singh, Jatinder K., Elastic-plastic transitional stress in a thin rotating disc with shaft having variable thickness under steady state temperature, Kragujevac Journal of Science 36 (2014) 5 -17. 
[42] Pankaj Thakur, Anupam S., Steady state thermal stresses in thin circular Disc by deformation with variable Load for different materials by Concept of Seth's Transition Theory, Mathematical Sciences International Journal 3 (1) (2014) 421-431. 